Centre interuniversitaire de recherche en économie quantitative

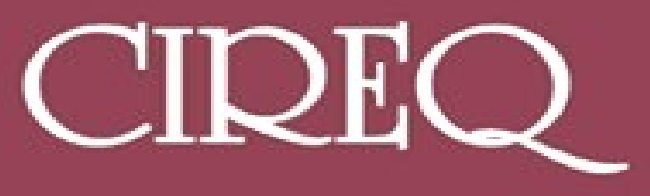

Cahier 20-2010

Product Filters, Acyclicity and Suzumura Consistency

Walter BOSSERT and Kotaro SUZUMURA 


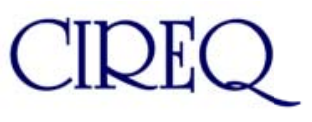

Le Centre interuniversitaire de recherche en économie quantitative (CIREQ) regroupe des chercheurs dans les domaines de l'économétrie, la théorie de la décision, la macroéconomie et les marchés financiers, la microéconomie appliquée et l'économie expérimentale ainsi que l'économie de l'environnement et des ressources naturelles. Ils proviennent principalement des universités de Montréal, McGill et Concordia. Le CIREQ offre un milieu dynamique de recherche en économie quantitative grâce au grand nombre d'activités qu'il organise (séminaires, ateliers, colloques) et de collaborateurs qu'il reçoit chaque année.

The Center for Interuniversity Research in Quantitative Economics (CIREQ) regroups researchers in the fields of econometrics, decision theory, macroeconomics and financial markets, applied microeconomics and experimental economics, and environmental and natural resources economics. They come mainly from the Université de Montréal, McGill University and Concordia University. CIREQ offers a dynamic environment of research in quantitative economics thanks to the large number of activities that it organizes (seminars, workshops, conferences) and to the visitors it receives every year.

\section{Cahier 20-2010}

\section{Product Filters, Acyclicity and Suzumura Consistency}

Walter BOSSERT and Kotaro SUZUMURA

CIREQ, Université de Montréal C.P. 6128, succursale Centre-ville Montréal (Québec) H3C 3J7 Canada cireq@umontreal.ca http://www.cireq.umontreal.ca
Universite th de Montréa
McGill

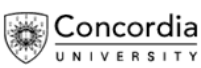


Ce cahier a également été publié par le Département de sciences économiques de l'Université de Montréal sous le numéro (2010-11).

This working paper was also published by the Department of Economics of the University of Montreal under number (2010-11).

Dépôt légal - Bibliothèque nationale du Canada, 2010, ISSN 0821-4441

Dépôt légal - Bibliothèque et Archives nationales du Québec, 2010

ISBN-13 : 978-2-89382-608-0 


\title{
Product Filters, Acyclicity and Suzumura Consistency*
}

\author{
WALter Bossert \\ Department of Economics and CIREQ \\ University of Montreal \\ P.O. Box 6128, Station Downtown \\ Montreal QC H3C 3J7 \\ Canada \\ FAX: (+1 514) 3437221 \\ e-mail: walter.bossert@umontreal.ca \\ and \\ Kotaro Suzumura \\ School of Political Science and Economics \\ Waseda University \\ 1-6-1 Nishi-Waseda \\ Shinjuku-ku, Tokyo 169-8050 \\ Japan \\ FAX: (+81 3) 52861818 \\ e-mail: k.suzumura@aoni.waseda.jp
}

This version: November 25, 2010

\footnotetext{
* Financial support from a Grant-in-Aid for Specially Promoted Research from the Ministry of Education, Culture, Sports, Science and Technology of Japan for the Project on Economic Analysis of Intergenerational Issues (grant number 22000001), the Fonds de Recherche sur la Société et la Culture of Québec, and the Social Sciences and Humanities Research Council of Canada is gratefully acknowledged.
} 


\begin{abstract}
In a seminal contribution, Hansson (1976) demonstrates that the collection of decisive coalitions associated with an Arrovian social welfare function forms an ultrafilter. He goes on to show that if transitivity is weakened to quasi-transitivity as the coherence property imposed on a social relation, the set of decisive coalitions is a filter. We examine the notion of decisiveness with acyclical or Suzumura consistent social preferences and without assuming that the social relation is complete. This leads to a new set-theoretic concept applied to product spaces. Journal of Economic Literature Classification Nos.: C02, D71.
\end{abstract}

Keywords: Product Filters, Decisiveness, Acyclicity, Suzumura Consistency. 


\section{Introduction}

Arrow's $(1951 ; 1963)$ theorem establishing the existence of a dictator as a consequence of a set of seemingly innocuous properties of a social welfare function is the most fundamental result in the theory of social choice. Its conclusion depends crucially on the assumption that the population under consideration is finite and alternative methods of proof provided by authors such as Fishburn (1970), Sen (1979) and Suzumura (2000) highlight the important role played by this finiteness property. Kirman and Sondermann (1972) and Hansson (1976) consider the structure of decisive coalitions in the Arrovian framework. A coalition (that is, a subset of the population) is decisive if its members can always guarantee a strict social preference for any alternative over any other if all coalition members have a strict preference for the former. Kirman and Sondermann (1972) and Hansson (1976) establish that, in the general case where the population may be finite or infinite, the set of decisive coalitions forms an ultrafilter, given that social relations are assumed to be orderings and Arrow's axioms unlimited domain, weak Pareto and independence of irrelevant alternatives are satisfied. In the finite population case, all ultrafilters are principal ultrafilters, that is, they are generated by a singleton. This singleton is, by definition of the decisiveness property, a dictator. Thus, the results of these two papers generate Arrow's theorem as a corollary. In contrast, if the population is infinite, there exist non-principal ultrafilters and these ultrafilters correspond to decisive coalition structures that are non-dictatorial. Kirman and Sondermann (1972) argue that sets of decisive coalitions that are non-principal ultrafilters still have a dictatorial flavor when expressed in a different space (leading to what they refer to as "invisible" dictators) but this does not make the underlying social welfare functions themselves dictatorial; see Hansson (1976) for a discussion.

There has been some renewed interest in specific applications of infinite population Arrovian social choice, particularly in the context of infinite horizon social choice problems where the unidirectional nature of the flow of time permits some natural domain restrictions; see, for instance, Ferejohn and Page (1978), Packel (1980) and Bossert and Suzumura (2010b). In this paper, we reexamine Hansson's (1976) approach from a different perspective by relaxing the properties imposed on social preferences. As in the original Arrovian setting, Hansson (1976) assumes in his first set of results that a collective choice rule always generates orderings. Moreover, he considers the case where social preferences are merely quasi-transitive but not necessarily transitive while retaining the richness properties of reflexivity and completeness. In this case, the family of decisive coalitions does 
not necessarily form an ultrafilter but it always is a filter. If the population is finite, the corresponding collective choice rules are oligarchies.

Bossert and Suzumura (2010a, Chapter 10) provide a comprehensive analysis of the resulting decisiveness structures when the requirements imposed on social relations are relaxed. If reflexivity and completeness are dropped, the decisive coalition structures resulting from Arrow's axioms are the same for transitivity and for quasi-transitivity: the family of decisive coalitions is a filter. As is the case for Hansson's (1976) results, the implications can be reversed in the sense that, for any given filter, there exists a collective choice rule that generates transitive (and thus quasi-transitive) social preferences that have this given filter as the family of decisive coalitions. A corollary of these observations is that reflexivity and completeness are redundant when social preferences are assumed to be quasi-transitive: the decisiveness structures are unchanged if these two richness properties are added.

In this paper, we consider an environment where social preferences are assumed to satisfy alternative weakenings of transitivity, namely, acyclicity or Suzumura consistency. Acyclicity rules out the existence of strict preference cycles, whereas Suzumura consistency (Suzumura, 1976) eliminates the existence of cycles with at least one strict preference. Transitivity implies Suzumura consistency which, in turn, implies acyclicity. Quasitransitivity is intermediate in strength between transitivity and acyclicity as well and it is independent of Suzumura consistency. In the case of a reflexive and complete relation, transitivity and Suzumura consistency are equivalent.

Suzumura consistency is an interesting property because it is necessary and sufficient for the existence of an ordering extension (Suzumura, 1976) - that is, a relation can be extended to an ordering respecting all weak and strict preferences of the underlying relation if and only if the original relation is Suzumura consistent. This fundamental insight represents a significant strengthening of the classical extension theorem established by Szpilrajn (1930) who showed that transitivity is sufficient for the existence of such an extension. Moreover, Suzumura consistency of a relation corresponds precisely to the requirement that an agent with such a relation is not a 'money pump' according to a wellknown illustration of 'incoherent' preferences (Raiffa, 1968, p.78). See Bossert (2008) for a brief overview of recent applications of Suzumura consistency.

Both acyclicity and Suzumura consistency are too weak to allow for the standard notion of decisive coalitions. A coalition cannot be decisive in the usual sense in this environment because its power to enforce a strict preference for an alternative over another may depend on the preferences of the remaining members of society. Thus, a notion of 
decisiveness that applies to pairs of coalitions has to be employed. Consider a pair of coalitions $\left(M, M^{\prime}\right)$. The coalition $M$ is said to be decisive against $M^{\prime}$ for a collective choice rule if and only if the two coalitions are disjoint and, for each possible preference profile, the social relation ranks an alternative $x$ as better than an alternative $y$ whenever the set of those who strictly prefer $x$ to $y$ is given by $M$ and the set of individuals who prefer $y$ to $x$ is $M^{\prime}$. We define what we call a product filter and show that the collection of pairs of coalitions with the above pairwise decisiveness structure is a product filter whenever social preferences are required to be acyclical and the collective choice rule respects the weak Pareto principle. Conversely, for any given product filter, it is possible to construct a collective choice rule that satisfies weak Pareto and neutrality and, moreover, produces Suzumura consistent social relations.

Two observations are worth pointing out at this stage. First, if social preferences are not required to be reflexive and complete, acyclicity and Suzumura consistency cannot be distinguished in terms of the pairwise decisive coalition structures they correspond to. This parallels the observations of Bossert and Suzumura (2010a, Chapter 10) regarding quasi-transitive and transitive social relations in the absence of reflexivity and completeness. The second (and, to us, more striking) observation is that a product filter structure allows us to generate collective choice rules that satisfy not only weak Pareto but also neutrality. Note that this property is not required (and neither is the weaker axiom of independence of irrelevant alternatives) when establishing that a product filter structure results from assuming weak Pareto along with the acyclicity of the social preferences.

In the following section, we introduce our basic definitions. A brief summary of Hansson's (1976) and Bossert and Suzumura's (2010a, Chapter 10) results is given in Section 3. Acyclicity and Suzumura consistency are analyzed in the Arrovian setting in Section 4. In particular, we introduce a pairwise notion of decisiveness and a new product filter concept. Section 5 provides a concluding discussion.

\section{Relations and collective choice rules}

Suppose there is an infinite set of alternatives $X$. We identify the population with the set $N$, which is assumed to contain at least two individuals. $N$ could be finite or infinite. A (binary) relation on $X$ is a subset $R$ of the Cartesian product $X \times X$. For notational convenience, we write $x R y$ instead of $(x, y) \in R$. A relation $R$ is asymmetric if and only if, for all $x, y \in X$,

$$
x R y \Rightarrow \neg y R x .
$$


The asymmetric part of a relation $R$ is denoted by $P$ and the symmetric part of $R$ is $I$. If $R$ is interpreted as a weak preference relation, $P$ is the strict preference relation corresponding to $R$ and $I$ is the indifference relation corresponding to $R$.

An ordering is a reflexive, complete and transitive relation. When $R$ is an ordering, we use chains such as, for instance, $x P y I z$ to indicate that each of the three alternatives $x, y, z \in X$ is indifferent to itself, $x$ is strictly preferred to $y$ and $z$, and $y$ and $z$ are indifferent.

A relation $R$ is quasi-transitive if and only if its asymmetric part $P$ is transitive, and $R$ is acyclical if and only if, for all $K \in \mathbb{N}$ and for all $x^{1}, \ldots, x^{K+1} \in X$,

$$
x^{k} P x^{k+1} \text { for all } k \in\{1, \ldots, K\} \Rightarrow \neg x^{K+1} P x^{1} .
$$

$R$ is Suzumura consistent if and only if, for all $K \in \mathbb{N}$ and for all $x^{1}, \ldots, x^{K+1} \in X$,

$$
x^{k} R x^{k+1} \text { for all } k \in\{1, \ldots, K\} \Rightarrow \neg x^{K+1} P x^{1} .
$$

Transitivity implies quasi-transitivity which, in turn, implies acyclicity. Analogously, transitivity implies Suzumura consistency, and Suzumura consistency implies acyclicity. Without further assumptions, the reverse implications are not true and, furthermore, quasi-transitivity and Suzumura consistency are independent. In the presence of reflexivity and completeness, transitivity and Suzumura consistency are equivalent.

It is assumed that each individual $n \in N$ ranks the elements of $X$ by means of an ordering $R_{n}$ with asymmetric part $P_{n}$ and symmetric part $I_{n}$. A profile is a list $\mathbf{R}=\left\langle R_{n}\right\rangle_{n \in N}$ of orderings on $X$, one for each member of society. The set of all such profiles is denoted by $\mathcal{R}^{N}$. For $\mathbf{R} \in \mathcal{R}^{N}$ and $x, y \in X$, the set of individuals who strictly prefer $x$ to $y$ in the profile $\mathbf{R}$ is $B(x, y ; \mathbf{R})=\left\{n \in N \mid x P_{n} y\right\}$.

$\mathcal{B}$ is set of all binary relations on $X$. A collective choice rule is a mapping $g: \mathcal{R}^{N} \rightarrow \mathcal{B}$. An unlimited domain assumption is built into the definition of a collective choice rule: $g$ is assumed to produce a social ranking $g(\mathbf{R})$ of the alternatives in $X$ for every possible profile $\mathbf{R} \in \mathcal{R}^{N}$. For simplicity of exposition, we use $R=g(\mathbf{R})$ to denote the social preference relation associated with the profile $\mathbf{R}$. The strict preference relation and the indifference relation corresponding to $R=g(\mathbf{R})$ are $P$ and $I . g$ is a Suzumura consistent collective choice rule if and only if $g(\mathbf{R})$ is Suzumura consistent for all $\mathbf{R} \in \mathcal{R}^{N}$, and $g$ is an acyclical collective choice rule if and only if $g(\mathbf{R})$ is acyclical for all $\mathbf{R} \in \mathcal{R}^{N}$. Analogously, $g$ is a quasi-transitive collective choice rule if and only if $g(\mathbf{R})$ is quasi-transitive for all $\mathbf{R} \in \mathcal{R}^{N}$, and $g$ is a transitive collective choice rule if and only if $g(\mathbf{R})$ is transitive for all $\mathbf{R} \in \mathcal{R}^{N} . g$ is a social welfare function if and only if $g(\mathbf{R})$ is an ordering for all $\mathbf{R} \in \mathcal{R}^{N}$. 
Arrow $(1951 ; 1963)$ imposed the axioms of unlimited domain, weak Pareto and independence of irrelevant alternatives and showed that, in the case of a finite population, the only social welfare functions that possess these three properties are dictatorial: there exists an individual such that, whenever this individual strictly prefers one alternative over another, this strict preference is reproduced in the social ranking, irrespective of the preferences of other members of society. As mentioned above, unlimited domain is already imposed by assuming that the domain of $g$ is given by $\mathcal{R}^{N}$. Weak Pareto is the standard unanimity property with respect to strict preferences.

Weak Pareto. For all $\mathbf{R} \in \mathcal{R}^{N}$ and for all $x, y \in X$,

$$
B(x, y ; \mathbf{R})=N \Rightarrow x P y
$$

where $R=g(\mathbf{R})$.

Independence of irrelevant alternatives demands that the social relative ranking of any two alternatives $x$ and $y$ for any profile $\mathbf{R}$ depends on the individual relative rankings of $x$ and $y$ in $\mathbf{R}$ only.

Independence of irrelevant alternatives. For all $\mathbf{R}, \mathbf{R}^{\prime} \in \mathcal{R}^{N}$ and for all $x, y \in X$,

$$
\left[x R_{n} y \Leftrightarrow x R_{n}^{\prime} y \text { and } y R_{n} x \Leftrightarrow y R_{n}^{\prime} x\right] \text { for all } n \in N \Rightarrow\left[x R y \Leftrightarrow x R^{\prime} y \text { and } y R x \Leftrightarrow y R^{\prime} x\right]
$$

where $R=g(\mathbf{R})$ and $R^{\prime}=g\left(\mathbf{R}^{\prime}\right)$.

A natural strengthening of this independence condition is neutrality. It requires that social rankings do not depend on the labels of the alternatives.

Neutrality. For all $\mathbf{R}, \mathbf{R}^{\prime} \in \mathcal{R}^{N}$ and for all $x, y, x^{\prime}, y^{\prime} \in X$, $\left[x R_{n} y \Leftrightarrow x^{\prime} R_{n}^{\prime} y^{\prime}\right.$ and $\left.y R_{n} x \Leftrightarrow y^{\prime} R_{n}^{\prime} x^{\prime}\right]$ for all $n \in N \Rightarrow\left[x R y \Leftrightarrow x^{\prime} R^{\prime} y^{\prime}\right.$ and $\left.y R x \Leftrightarrow y^{\prime} R^{\prime} x^{\prime}\right]$ where $R=g(\mathbf{R})$ and $R^{\prime}=g\left(\mathbf{R}^{\prime}\right)$.

\section{Decisiveness, filters and ultrafilters}

Let $g$ be a collective choice rule. A set of individuals $M \subseteq N$ (also referred to as a coalition) is decisive for $g$ if and only if, for all $\mathbf{R} \in \mathcal{R}^{N}$ and for all $x, y \in X$,

$$
M \subseteq B(x, y ; \mathbf{R}) \Rightarrow x P y .
$$


The grand coalition $N$ is decisive for any collective choice rule satisfying weak Pareto. If there exists an individual $n \in N$ such that $\{n\}$ is decisive for $g$, individual $n$ is a dictator for $g$. Let $\mathcal{D}_{g}$ denote the set of all decisive coalitions for a collective choice rule $g$.

Weaker forms of decisiveness have been considered in the literature. Capitalizing on Arrow (1951, p.53), Sen (1970, p.42) defines a coalition $M \subseteq N$ to be almost decisive for $g$ if and only if, for all $\mathbf{R} \in \mathcal{R}^{N}$ and for all $x, y \in X$,

$$
[B(x, y ; \mathbf{R})=M \text { and } B(y, x ; \mathbf{R})=N \backslash M] \Rightarrow x P y .
$$

If a coalition $M$ is decisive for $g$, it follows that $M$ is almost decisive for $g$; this implication is a direct consequence of the conditional nature of the decisiveness power expressed in the above definition. Another class of conditional decisiveness notions, labeled potential decisiveness, appears in Sen (1976, p.240).

A filter on $N$ is a collection $\mathcal{F}$ of subsets of $N$ such that

f.1. $\emptyset \notin \mathcal{F}$;

f.2. $N \in \mathcal{F}$;

f.3. for all $M, M^{\prime} \in \mathcal{F}, M \cap M^{\prime} \in \mathcal{F}$;

f.4. for all $M, M^{\prime} \subseteq N,\left[\left[M \in \mathcal{F}\right.\right.$ and $\left.\left.M \subseteq M^{\prime}\right] \Rightarrow M^{\prime} \in \mathcal{F}\right]$.

An ultrafilter on $N$ is a collection $\mathcal{U}$ of subsets of $N$ such that

u.1. $\emptyset \notin \mathcal{U}$;

u.2. for all $M \subseteq N,[M \in \mathcal{U}$ or $N \backslash M \in \mathcal{U}]$;

u.3. for all $M, M^{\prime} \in \mathcal{U}, M \cap M^{\prime} \in \mathcal{U}$.

Every ultrafilter is a filter as is shown for instance, in Bossert and Suzumura (2010a, Chapter 2). The reverse statement is not true.

An ultrafilter $\mathcal{U}$ is principal if there exists $n \in N$ such that, for all $M \subseteq N, M \in \mathcal{U}$ if and only if $n \in M$. In this case, we say that $\mathcal{U}$ is generated by the singleton $\{n\}$. Otherwise, $\mathcal{U}$ is a free ultrafilter. If $N$ is a finite set, then all ultrafilters are principal. If $N$ is infinite, however, there also exist free ultrafilters but they cannot be defined explicitly; the available proofs of their existence are non-constructive and rely on the axiom of choice or similar properties.

Hansson (1976) has shown that if a social welfare function $g$ satisfies weak Pareto and independence of irrelevant alternatives, then $\mathcal{D}_{g}$ must be an ultrafilter on $N$. Conversely, if $\mathcal{U}$ is an ultrafilter on $N$, then there exists a social welfare function $g$ satisfying weak Pareto and independence of irrelevant alternatives such that $\mathcal{D}_{g}=\mathcal{U}$. 
Hansson's (1976) results allow us to obtain Arrow's $(1951 ; 1963)$ theorem for the special case of a finite population: if the set of decisive coalitions contains a singleton $\{n\}$, individual $n$ is a dictator. Because the set of decisive coalitions is an ultrafilter and all ultrafilters are principal if $N$ is finite, there exists an individual $n$ who is a dictator. In the infinite population case, a set of decisive coalitions that is a principal ultrafilter corresponds to a dictatorship just as in the finite case. However, because not all ultrafilters are principal if $N$ is infinite, Arrow's axioms allow for non-dictatorial social welfare functions - namely, those whose sets of decisive coalitions correspond to free ultrafilters.

Hansson (1976) also examined collective choice rules with social relations that are merely required to be quasi-transitive rather than transitive (but he retained the reflexivity and completeness assumptions). In that case, the set of decisive coalitions $\mathcal{D}_{g}$ is not necessarily an ultrafilter but it still is a filter whenever $g$ satisfies weak Pareto and independence of irrelevant alternatives. In analogy to the corresponding observation for ultrafilters, for any filter $\mathcal{F}$, there exists a collective choice rule $g$ that yields reflexive, complete and quasi-transitive social relations and satisfies weak Pareto and independence of irrelevant alternatives such that $\mathcal{D}_{g}=\mathcal{F}$. When the population is finite, filters correspond to collections of decisive coalitions that are oligarchies.

Bossert and Suzumura (2010a, Chapter 10) reexamine Hansson's (1976) observations involving transitive and quasi-transitive social relations satisfying the Arrow axioms in the absence of reflexivity and completeness. Although the resulting decisiveness structures can be recovered employing steps analogous to those that appear in Hansson (1976), there is an interesting difference: once reflexivity and completeness are dropped, the families of decisive coalitions associated with transitive collective choice rules and with quasitransitive collective choice rules can no longer be distinguished. Intuitively, this is the case because only strict preferences are imposed by weak Pareto and, in the absence of completeness, an absence of strict preference does not imply a weak preference in the reverse direction. Moreover, as a corollary to these results, quasi-transitivity with reflexivity and completeness is equivalent to quasi-transitivity without reflexivity and completeness, whereas transitivity without reflexivity and completeness results in a weaker structural property of the family of decisive coalitions - namely, the same structure that obtains for quasi-transitivity. It is worth noting that decisive coalitions continue to be well-defined; this is in contrast with the structure to be uncovered in the following section where we consider acyclical and Suzumura consistent social preferences. Bossert and Suzumura (2010a, Chapter 10) establish that if a quasi-transitive collective choice rule $g$ satisfies weak Pareto and independence of irrelevant alternatives, then $\mathcal{D}_{g}$ must be a filter 
on $N$. In addition, they show that, for any filter $\mathcal{F}$ on $N$, there exists a transitive collective choice rule $g$ that satisfies weak Pareto and independence of irrelevant alternatives such that $\mathcal{D}_{g}=\mathcal{F}$.

\section{Pairwise decisiveness and product filters}

If, instead of transitivity or quasi-transitivity, acyclicity or Suzumura consistency is imposed on social preferences, the approach involving decisive coalitions and filters summarized in the previous section must be amended. This is the case because the existence of such coalitions is not guaranteed. A coalition cannot be decisive in the usual sense if a social preference relation is required to be merely acyclical or Suzumura consistent because its power to enforce a strict preference for an alternative over another may depend on the preferences of the remaining members of society. To deal with this observation, we introduce a new set-theoretic concept that applies to pairs of coalitions - that is, we formulate a filter structure for a product space.

Our new definition of decisiveness is a property of a pair of coalitions $\left(M, M^{\prime}\right)$ with $M, M^{\prime} \subseteq N$. We say that $M$ is decisive against $M^{\prime}$ for a collective choice rule $g$ if and only if the two coalitions are disjoint and the social relation declares $x$ to be strictly preferred to $y$ whenever $B(x, y ; \mathbf{R})=M$ and $B(y, x ; \mathbf{R})=M^{\prime}$. The set of pairs of coalitions $\left(M, M^{\prime}\right)$ with this property for a collective choice rule $g$ is denoted by $\mathcal{A}_{g}$. That is, for all $M, M^{\prime} \subseteq N$, we let $\left(M, M^{\prime}\right) \in \mathcal{A}_{g}$ if and only if

a.1. $M \cap M^{\prime}=\emptyset$

and

a.2. for all $\mathbf{R} \in \mathcal{R}^{N}$ and for all $x, y \in X$,

$$
\left[B(x, y ; \mathbf{R})=M \text { and } B(y, x ; \mathbf{R})=M^{\prime}\right] \Rightarrow x P y .
$$

Clearly, if $g$ satisfies weak Pareto, then the pair $(N, \emptyset)$ is in $\mathcal{A}_{g}$.

There is a connection between this new notion of pairwise decisiveness and Sen's (1970) almost decisive coalitions mentioned in the previous section: if $M \subseteq N$ is almost decisive for $g$, then $M$ is decisive against $N \backslash M$ for $g$. However, the definition of an almost decisive coalition is silent about any decisiveness properties involving pairs $\left(M, M^{\prime}\right)$ such that $M^{\prime}$ is not equal to the complement of $M$ in $N$.

A product filter on $N \times N$ is a collection $\mathcal{W}$ of pairs of subsets of $N$ such that 
w.1. $(N, \emptyset) \in \mathcal{W}$

w.2. for all $\left(M, M^{\prime}\right) \in \mathcal{W}, M \cap M^{\prime}=\emptyset$;

w.3. for all $K \in \mathbb{N}$ and for all $M^{1}, \ldots, M^{K}, \bar{M}^{1}, \ldots, \bar{M}^{K} \subseteq N$,

$$
\left(M^{k}, \bar{M}^{k}\right) \in \mathcal{W} \quad \text { for all } k \in\{1, \ldots, K\} \Rightarrow\left(\cup_{j=1}^{K} \bar{M}^{j}, \cup_{j=1}^{K} M^{j}\right) \notin \mathcal{W} .
$$

There is a close conceptual link between filters and ultrafilters, on one hand, and product filters, on the other. In particular, any filter on $N$ (and, thus, any ultrafilter on $N$ ) induces a product filter on $N \times N$ in the following sense. Suppose $\mathcal{F}$ is a filter on $N$. Define the collection $\mathcal{W}_{\mathcal{F}}^{0}$ of pairs of subsets of $N \times N$ by letting

$$
\mathcal{W}_{\mathcal{F}}^{0}=\left\{\left(M, M^{\prime}\right) \mid M \in \mathcal{F} \text { and } M^{\prime} \subseteq N \text { and } M \cap M^{\prime}=\emptyset\right\}
$$

To prove that $\mathcal{W}_{\mathcal{F}}^{0}$ satisfies $w .1$, note first that, by $f .2, N \in \mathcal{F}$. Furthermore, $\emptyset \subseteq N$ and $N \cap \emptyset=\emptyset$. Thus, by definition of $\mathcal{W}_{\mathcal{F}}^{0}$, it follows that $(N, \emptyset) \in \mathcal{W}_{\mathcal{F}}^{0}$. $w .2$ is an immediate consequence of the definition of $\mathcal{W}_{\mathcal{F}}^{0}$. Finally, to establish $w .3$, suppose that $K \in \mathbb{N}$ and $M^{1}, \ldots, M^{K}, \bar{M}^{1}, \ldots, \bar{M}^{K} \subseteq N$ are such that

$$
\left(M^{k}, \bar{M}^{k}\right) \in \mathcal{W}_{\mathcal{F}}^{0} \quad \text { for all } k \in\{1, \ldots, K\} .
$$

By definition of $\mathcal{W}_{\mathcal{F}}^{0}$, this is equivalent to

$$
M^{k} \in \mathcal{F} \quad \text { and } \quad \bar{M}^{k} \subseteq N \text { and } \quad M^{k} \cap \bar{M}^{k}=\emptyset \text { for all } k \in\{1, \ldots, K\} .
$$

If $\left(\cup_{j=1}^{K} \bar{M}^{j}, \cup_{j=1}^{K} M^{j}\right) \in \mathcal{W}_{\mathcal{F}}^{0}$, we have

$$
\cup_{j=1}^{K} \bar{M}^{j} \in \mathcal{F} \quad \text { and } \quad \cup_{j=1}^{K} M^{j} \subseteq N \quad \text { and } \quad\left(\cup_{j=1}^{K} \bar{M}^{j}\right) \cap\left(\cup_{j=1}^{K} M^{j}\right)=\emptyset .
$$

By f.3, $\left(\cup_{j=1}^{K} \bar{M}^{j}\right) \cap M^{k} \in \mathcal{F}$ for any $k \in\{1, \ldots, K\}$. But $\left(\cup_{j=1}^{K} \bar{M}^{j}\right) \cap M^{k}=\emptyset$ for all $k \in\{1, \ldots, K\}$ because $\left(\cup_{j=1}^{K} \bar{M}^{j}\right) \cap\left(\cup_{j=1}^{K} M^{j}\right)=\emptyset$. This contradicts $f .1$.

Note that $f .4$ is not employed in the above argument. Thus, even a 'weak filter' structure that is obtained by dropping requirement $f .4$ is sufficient to induce a product filter in the above described manner.

A few examples may be helpful in illustrating product filters and their relation to filters and ultrafilters.

Example 1 Suppose $1 \in N$ and let

$$
\mathcal{W}^{1}=\left\{\left(M, M^{\prime}\right) \mid 1 \in M \subseteq N \text { and } M^{\prime} \subseteq N \backslash M\right\} .
$$

This is a product filter on $N \times N$ that can be induced by the principal ultrafilter $\mathcal{U}^{1}$ on $N$ generated by the singleton $\{1\}$, that is, by $\mathcal{U}^{1}=\{M \subseteq N \mid 1 \in M\}$. 
Example 2 Let

$$
\mathcal{W}^{2}=\{(N, \emptyset)\}
$$

That $\mathcal{W}^{2}$ is a product filter on $N \times N$ is straightforward to verify. Moreover, it is easy to see that $\mathcal{W}^{2}$ is induced by the filter on $N$ given by $\mathcal{F}^{2}=\{N\}$.

Example 3 Suppose that $1 \in N$ and define

$$
\mathcal{W}^{3}=\{(N, \emptyset)\} \cup\left\{\left(\{1\}, M^{\prime}\right) \mid M^{\prime} \subseteq N \backslash\{1\}\right\} .
$$

This is a product filter on $N \times N$ that is induced by the collection $\{N,\{1\}\}$. This collection is an example of a 'weak filter' on $N$-that is, a collection of subsets of $N$ that satisfies f.1, f.2 and f.3.

Example 4 Suppose 1,2,3 $N$ and let

$$
\mathcal{W}^{4}=\{(N, \emptyset),(\{1,2\}, N \backslash\{1,2\}),(\{1,3\}, N \backslash\{1,3\})\} .
$$

It is straightforward to verify that this collection possesses the properties of a product filter on $N \times N$.

We now obtain the following two theorems. When combined, they characterize acyclical (or Suzumura consistent) unanimous and neutral social choice in terms of the resulting pairwise decisiveness structure. As mentioned in the introduction, neutrality is not required in the first result and it follows in the second theorem as a consequence of the product filter assumption.

Theorem 1 If an acyclical collective choice rule $g$ satisfies weak Pareto, then $\mathcal{A}_{g}$ is a product filter on $N \times N$.

Proof. Suppose $g$ is an acyclical collective choice rule that satisfies weak Pareto. We prove that $\mathcal{A}_{g}$ possesses the three properties of a product filter.

w.1. That $(N, \emptyset) \in \mathcal{A}_{g}$ follows from weak Pareto.

$w .2$. This property is an immediate consequence of $a .1$.

$w .3$. By way of contradiction, suppose that $w .3$ is violated. Then there exist $K \in \mathbb{N}$ and $M^{1}, \ldots, M^{K}, \bar{M}^{1}, \ldots, \bar{M}^{K} \subseteq N$ such that

$$
\left(M^{k}, \bar{M}^{k}\right) \in \mathcal{A}_{g} \text { for all } k \in\{1, \ldots, K\} \quad \text { and } \quad\left(\cup_{j=1}^{K} \bar{M}^{j}, \cup_{j=1}^{K} M^{j}\right) \in \mathcal{A}_{g} .
$$


Let $x^{1}, \ldots, x^{K+1} \in X$ and construct a profile $\mathbf{R} \in \mathcal{R}^{N}$ as follows. For all $m \in \cup_{j=1}^{K} M^{j}$, let

$$
x^{1} R_{m} \ldots R_{m} x^{K+1}
$$

where, for all $k \in\{1, \ldots, K\}$,

$$
\left[x^{k} P_{m} x^{k+1} \Leftrightarrow m \in M^{k}\right] \text { and }\left[x^{k} I_{m} x^{k+1} \Leftrightarrow m \notin M^{k}\right] .
$$

Furthermore, for all $m \in \cup_{j=1}^{K} \bar{M}^{j}$, let

$$
x^{K+1} R_{m} \ldots R_{m} x^{1}
$$

where, for all $k \in\{1, \ldots, K\}$,

$$
\left[x^{k+1} P_{m} x^{k} \Leftrightarrow m \in \bar{M}^{k}\right] \text { and }\left[x^{k+1} I_{m} x^{k} \Leftrightarrow m \notin \bar{M}^{k}\right] .
$$

To complete the definition of the preferences of the individuals in $\left(\cup_{j=1}^{K} M^{j}\right) \cup\left(\cup_{j=1}^{K} \bar{M}^{j}\right)$, let

$$
x P_{m} y I_{m} z
$$

for all $m \in\left(\cup_{j=1}^{K} M^{j}\right) \cup\left(\cup_{j=1}^{K} \bar{M}^{j}\right)$, for all $x \in\left\{x^{1}, \ldots, x^{K+1}\right\}$ and for all $y, z \in$ $X \backslash\left\{x^{1}, \ldots, x^{K+1}\right\}$.

For all $m \in N \backslash\left(\left(\cup_{j=1}^{K} M^{j}\right) \cup\left(\cup_{j=1}^{K} \bar{M}^{j}\right)\right)$ and for all $x, y \in X$, let

$$
x I_{m} y .
$$

Clearly, the profile thus defined consists of individual orderings. By definition,

$$
B\left(x^{k}, x^{k+1} ; \mathbf{R}\right)=M^{k} \quad \text { and } \quad B\left(x^{k+1}, x^{k} ; \mathbf{R}\right)=\bar{M}^{k}
$$

for all $k \in\{1, \ldots, K\}$. Thus, because $\left(M^{k}, \bar{M}^{k}\right) \in \mathcal{A}_{g}$ by assumption,

$$
x^{k} P x^{k+1} \text { for all } k \in\{1, \ldots, K\} .
$$

Because each member of $\cup_{j=1}^{K} M^{j}$ belongs to at least one $M^{k}$ (and, thus, strictly prefers $x^{k}$ to $\left.x^{k+1}\right)$, (1) together with the transitivity of individual preferences implies

$$
x^{1} P_{m} x^{K+1}
$$

for all $m \in \cup_{j=1}^{K} M^{j}$. Analogously, because each member of $\cup_{j=1}^{K} \bar{M}^{j}$ belongs to at least one $\bar{M}^{k}$ (and, thus, strictly prefers $x^{k+1}$ to $x^{k}$ ), (2) together with the transitivity of individual preferences implies

$$
x^{K+1} P_{m} x^{1}
$$


for all $m \in \cup_{j=1}^{K} \bar{M}^{j}$. Finally, it is clear that

$$
x^{1} I_{m} x^{K+1}
$$

for all $m \in N \backslash\left(\left(\cup_{j=1}^{K} M^{j}\right) \cup\left(\cup_{j=1}^{K} \bar{M}^{j}\right)\right)$ and, thus,

$$
B\left(x^{K+1}, x^{1} ; \mathbf{R}\right)=\cup_{j=1}^{K} \bar{M}^{j} \quad \text { and } \quad B\left(x^{1}, x^{K+1} ; \mathbf{R}\right)=\cup_{j=1}^{K} M^{j} .
$$

By assumption, $\left(\cup_{j=1}^{K} \bar{M}^{j}, \cup_{j=1}^{K} M^{j}\right) \in \mathcal{A}_{g}$ and, thus,

$$
x^{K+1} P x^{1}
$$

which, together with (3), contradicts the acyclicity of $R=g(\mathbf{R})$.

Theorem 2 For any product filter $\mathcal{W}$ on $N \times N$, there exists a Suzumura consistent collective choice rule $g$ satisfying weak Pareto and neutrality such that $\mathcal{A}_{g}=\mathcal{W}$.

Proof. Let $\mathcal{W}$ be a product filter on $N \times N$. Define a collective choice rule $g$ by letting, for all $\mathbf{R} \in \mathcal{R}^{N}$ and for all $x, y \in X$,

$$
x R y \Leftrightarrow(B(x, y ; \mathbf{R}), B(y, x ; \mathbf{R})) \in \mathcal{W} .
$$

As an auxiliary result, we show that $R=g(\mathbf{R})$ is asymmetric for all profiles $\mathbf{R} \in \mathcal{R}^{N}$. By way of contradiction, suppose that there exist $\mathbf{R} \in \mathcal{R}^{N}$ and $x, y \in X$ such that $x R y$ and $y R x$. By definition of $g$,

$$
(B(x, y ; \mathbf{R}), B(y, x ; \mathbf{R})) \in \mathcal{W} \quad \text { and } \quad(B(y, x ; \mathbf{R}), B(x, y ; \mathbf{R})) \in \mathcal{W} .
$$

Letting $K=1, M^{1}=B(x, y ; \mathbf{R})$ and $\bar{M}^{1}=B(y, x ; \mathbf{R})$, it follows that

$$
\left(M^{1}, \bar{M}^{1}\right) \in \mathcal{W} \quad \text { and } \quad\left(\bar{M}^{1}, M^{1}\right)=\left(\cup_{j=1}^{K} \bar{M}^{j}, \cup_{j=1}^{K} M^{j}\right) \in \mathcal{W},
$$

contradicting $w .3$.

That $g$ satisfies weak Pareto follows from $w \cdot 1,(4)$ and the asymmetry of $R=g(\mathbf{R})$.

To prove that $g$ is neutral, suppose that $\mathbf{R}, \mathbf{R}^{\prime} \in \mathcal{R}^{N}$ and $x, y, x^{\prime}, y^{\prime} \in X$ are such that

$$
\left[x R_{n} y \Leftrightarrow x^{\prime} R_{n}^{\prime} y^{\prime} \text { and } y R_{n} x \Leftrightarrow y^{\prime} R_{n}^{\prime} x^{\prime}\right] \text { for all } n \in N \text {. }
$$

This implies that $B(x, y ; \mathbf{R})=B\left(x^{\prime}, y^{\prime} ; \mathbf{R}^{\prime}\right)$ and $B(y, x ; \mathbf{R})=B\left(y^{\prime}, x^{\prime} ; \mathbf{R}^{\prime}\right)$. Hence, using (4), we obtain

$$
\begin{aligned}
x R y & \Leftrightarrow(B(x, y ; \mathbf{R}), B(y, x ; \mathbf{R})) \in \mathcal{W} \\
& \Leftrightarrow\left(B\left(x^{\prime}, y^{\prime} ; \mathbf{R}^{\prime}\right), B\left(y^{\prime}, x^{\prime} ; \mathbf{R}^{\prime}\right)\right) \in \mathcal{W} \\
& \Leftrightarrow x^{\prime} R^{\prime} y^{\prime}
\end{aligned}
$$


and

$$
\begin{aligned}
y R x & \Leftrightarrow(B(y, x ; \mathbf{R}), B(x, y ; \mathbf{R})) \in \mathcal{W} \\
& \Leftrightarrow\left(B\left(y^{\prime}, x^{\prime} ; \mathbf{R}^{\prime}\right), B\left(x^{\prime}, y^{\prime} ; \mathbf{R}^{\prime}\right)\right) \in \mathcal{W} \\
& \Leftrightarrow y^{\prime} R^{\prime} x^{\prime} .
\end{aligned}
$$

We now show that $R=g(\mathbf{R})$ is Suzumura consistent for all possible profiles. Let $\mathbf{R} \in \mathcal{R}^{N}, K \in \mathbb{N}$ and $x^{1}, \ldots, x^{K+1} \in X$, and suppose that

$$
x^{k} R x^{k+1} \text { for all } k \in\{1, \ldots, K\} .
$$

By (4) and the asymmetry of $R,(5)$ is equivalent to

$$
\left(B\left(x^{k}, x^{k+1} ; \mathbf{R}\right), B\left(x^{k+1}, x^{k} ; \mathbf{R}\right)\right) \in \mathcal{W} \text { for all } k \in\{1, \ldots, K\} .
$$

Let $M^{k}=B\left(x^{k}, x^{k+1} ; \mathbf{R}\right)$ and $\bar{M}^{k}=B\left(x^{k+1}, x^{k} ; \mathbf{R}\right)$ for all $k \in\{1, \ldots, K\}$. It follows that, for all $k \in\{1, \ldots, K\}$ and for all $m \in \cup_{j=1}^{K} M^{j}$,

$$
\left[x^{k} P_{m} x^{k+1} \Leftrightarrow m \in M^{k}\right] \text { and }\left[x^{k} I_{m} x^{k+1} \Leftrightarrow m \notin M^{k}\right] .
$$

Analogously, for all $k \in\{1, \ldots, K\}$ and for all $m \in \cup_{j=1}^{K} \bar{M}^{j}$,

$$
\left[x^{k+1} P_{m} x^{k} \Leftrightarrow m \in \bar{M}^{k}\right] \text { and }\left[x^{k+1} I_{m} x^{k} \Leftrightarrow m \notin \bar{M}^{k}\right] .
$$

Because individual preferences are orderings and each member of $\cup_{j=1}^{K} M^{j}$ appears in at least one $M^{k}$ and, analogously, each member of $\cup_{j=1}^{K} \bar{M}^{j}$ appears in at least one $\bar{M}^{k}$, we obtain

$$
\left[x^{1} P_{m} x^{K+1} \text { for all } m \in \cup_{j=1}^{K} M^{j}\right] \text { and }\left[x^{K+1} P_{m} x^{1} \text { for all } m \in \cup_{j=1}^{K} \bar{M}^{j}\right] .
$$

By definition of $R$ and using the transitivity of individual preferences again, it follows that $x^{1} I_{m} x^{K+1}$ for all $m \in N \backslash\left(\left(\cup_{j=1}^{K} M^{j}\right) \cup\left(\cup_{j=1}^{K} \bar{M}^{j}\right)\right)$ and, therefore,

$$
B\left(x^{K+1}, x^{1} ; \mathbf{R}\right)=\cup_{j=1}^{K} \bar{M}^{j} \quad \text { and } \quad B\left(x^{1}, x^{K+1} ; \mathbf{R}\right)=\cup_{j=1}^{K} M^{j} .
$$

By $w .3,\left(\cup_{j=1}^{K} \bar{M}^{j}, \cup_{j=1}^{K} M^{j}\right) \notin \mathcal{W}$ and, thus,

$$
\neg x^{K+1} R x^{1}
$$

by (4). Because $R$ is asymmetric, this is equivalent to

$$
\neg x^{K+1} P x^{1} .
$$


Thus, $R$ is Suzumura consistent.

It remains to show that $\mathcal{A}_{g}=\mathcal{W}$.

Suppose first that $\left(M, M^{\prime}\right) \in \mathcal{A}_{g}$. By $a .1, M \cap M^{\prime}=\emptyset$. Let $\mathbf{R} \in \mathcal{R}^{N}$ and $x, y \in X$ be such that

$$
B(x, y ; \mathbf{R})=M \quad \text { and } \quad B(y, x ; \mathbf{R})=M^{\prime} .
$$

Because $M$ is decisive against $M^{\prime}$ for $g$, it follows that $x P y$ and hence $x R y$. By (4), $\left(M, M^{\prime}\right) \in \mathcal{W}$.

Finally, suppose that $\left(M, M^{\prime}\right) \in \mathcal{W}$. By $w .2, M \cap M^{\prime}=\emptyset$. Let $\mathbf{R} \in \mathcal{R}^{N}$ and $x, y \in X$ be such that

$$
B(x, y ; \mathbf{R})=M \quad \text { and } \quad B(y, x ; \mathbf{R})=M^{\prime} .
$$

By (4), $x R y$ and hence $x P y$ because $R$ is asymmetric. Thus, $M$ is decisive against $M^{\prime}$ for $g$, that is, $\left(M, M^{\prime}\right) \in \mathcal{A}_{g}$.

We now return to the examples presented earlier in this section and illustrate some collective choice rules that can be associated with the requisite product filters.

The product filter of Example 1 can be generated by a dictatorship of individual 1 defined by letting $g^{1}: \mathcal{R}^{N} \rightarrow \mathcal{B}$ be such that, for all $\mathbf{R} \in \mathcal{R}^{N}$ and for all $x, y \in X$,

$$
x R^{1} y \Leftrightarrow 1 \in B(x, y ; \mathbf{R}) .
$$

In Example 2, the Pareto (or unanimity) rule can be employed to induce $(N, \emptyset)$ as the only member of the requisite product filter. Formally, let $g^{2}: \mathcal{R}^{N} \rightarrow \mathcal{B}$ be such that, for all $\mathbf{R} \in \mathcal{R}^{N}$ and for all $x, y \in X$,

$$
x R^{2} y \Leftrightarrow B(x, y ; \mathbf{R})=N
$$

The product filter of Example 3 can be obtained from a collective choice rule such that an alternative $x$ is socially preferred to an alternative $y$ if either individual 1 strictly prefers $x$ to $y$ or there is unanimity regarding the strict preference for $x$ over $y$. This example combines an extremely asymmetric collective choice rule (a dictatorship) with an extremely symmetric rule (the unanimity rule). Let $g^{3}: \mathcal{R}^{N} \rightarrow \mathcal{B}$ be such that, for all $\mathbf{R} \in \mathcal{R}^{N}$ and for all $x, y \in X$,

$$
x R^{3} y \Leftrightarrow B(x, y ; \mathbf{R})=\{1\} \text { or } B(x, y ; \mathbf{R})=N .
$$

Example 3 illustrates that if quasi-transitivity is weakened to Suzumura consistency, the set of possible rules that is obtained becomes considerably more rich. 
A collective choice rule generating the product filter of Example 4 can be obtained by letting $g^{4}: \mathcal{R}^{N} \rightarrow \mathcal{B}$ be such that, for all $\mathbf{R} \in \mathcal{R}^{N}$ and for all $x, y \in X$,

$$
\begin{aligned}
x R^{4} y \Leftrightarrow \quad B(x, y ; \mathbf{R}) & =\{1,2\} \text { and } B(y, x ; \mathbf{R})=N \backslash\{1,2\} \text { or } \\
B(x, y ; \mathbf{R}) & =\{1,3\} \text { and } B(y, x ; \mathbf{R})=N \backslash\{1,3\} \text { or } \\
B(x, y ; \mathbf{R}) & =N .
\end{aligned}
$$

The definition of $g^{4}$ makes the decisiveness power of the coalitions $\{1,2\}$ and $\{1,3\}$ conditional on the preferences of the other members of society: only if the entire complement of $\{1,2\}$ (of $\{1,3\}$, respectively) in $N$ strictly prefers $y$ to $x$ and 1 and 2 ( 1 and 3 , respectively) strictly prefer $x$ to $y$ does $g^{4}$ declare $x$ to be socially better than $y$. For that reason, Example 4 illustrates a product filter that cannot be induced by a filter or a 'weak filter' employing the method outlined prior to the examples.

\section{Conclusion}

To the best of our knowledge, the notions of pairwise decisiveness and product filters developed in the previous section have not appeared in the earlier literature. These new structures appear to deserve further analysis. In particular, it might be of interest to examine possible applications of product filters in areas other than collective decision making.

As mentioned earlier, collective choice rules based on sets of decisive coalitions that form ultrafilters or filters lead to straightforward special cases if the population is finite: in essence, ultrafilters are associated with dictatorships, filters correspond to oligarchies. There does not appear to be an easily identifiable reduction of possibilities where product filter are concerned. As Examples 3 and 4 illustrate, there exists a variety of collective choice rules that can be defined explicitly regardless of whether the population is finite or infinite.

The results of this paper are established in a general framework where attention is restricted to the axioms employed in Arrow's impossibility theorem and neutrality. There have been approaches that examine to what extent weakenings of the requirements imposed on social relations allow us to obtain collective choice rules that may have additional properties such as anonymity or compliance with the strong rather than merely the weak Pareto principle; see, in particular, Sen (1969; 1970), Weymark (1984), Bossert and Suzumura (2008). Bossert ad Suzumura (2010c) analyze social relations that are both quasi-transitive and Suzumura consistent. 


\section{References}

Arrow, K.J. (1951, second ed. 1963), Social Choice and Individual Values, Wiley, New York.

Bossert, W. (2008), Suzumura consistency, in: P.K. Pattanaik, K. Tadenuma, Y. Xu and N. Yoshihara (eds.), Rational Choice and Social Welfare: Theory and Applications, Springer, Berlin, 159-179.

Bossert, W. and K. Suzumura (2008), A characterization of consistent collective choice rules, Journal of Economic Theory 138, 311-320; Erratum in 140, 355.

Bossert, W. and K. Suzumura (2010a), Consistency, Choice, and Rationality, Harvard University Press, Cambridge, MA.

Bossert, W. and K. Suzumura (2010b), Multi-profile intergenerational social choice, Social Choice and Welfare, forthcoming.

Bossert, W. and K. Suzumura (2010c), Quasi-transitive and Suzumura consistent relations, CIREQ Working Paper, University of Montreal.

Ferejohn, J. and T. Page (1978), On the foundations of intertemporal choice, American Journal of Agricultural Economics 60, 269-275.

Fishburn, P.C. (1970), Arrow's impossibility theorem: concise proof and infinite voters, Journal of Economic Theory 2, 103-106.

Hansson, B. (1976), The existence of group preference functions, Public Choice 38, 89-98.

Kirman, A.P. and D. Sondermann (1972), Arrow's theorem, many agents, and invisible dictators, Journal of Economic Theory 5, 267-277.

Packel, E. (1980), Impossibility results in the axiomatic theory of intertemporal choice, Public Choice 35, 219-227.

Raiffa, H. (1968), Decision Analysis, Addison-Wesley, Reading, MA.

Sen, A.K. (1969), Quasi-transitivity, rational choice and collective decisions, Review of Economic Studies 36, 381-393.

Sen, A.K. (1970), Collective Choice and Social Welfare, Holden-Day, San Francisco. 
Sen, A.K. (1976), Liberty, unanimity and rights, Economica 43, 217-245.

Sen, A.K. (1979), Personal utilities and public judgements: or what's wrong with welfare economics?, Economic Journal 89, 537-558.

Suzumura, K. (1976), Remarks on the theory of collective choice, Economica 43, 381390.

Suzumura, K. (2000), Welfare economics beyond welfarist-consequentialism, Japanese Economic Review 51, 1-32.

Szpilrajn, E. (1930), Sur l'extension de l'ordre partiel, Fundamenta Mathematicae 16, 386-389.

Weymark, J.A. (1984), Arrow's theorem with social quasi-orderings," Public Choice 42, 235-246. 